\section{Visible light-induced crosslink- ing of unmodified gelatin with PEGDA for DLP-3D printable hydrogels}

\author{
Michael Zanon, ${ }^{1}$ Desirée Baruffaldi,, 1,2 \\ Marco Sangermano, ${ }^{1}$ Candido Fabrizio \\ Pirri,1,2 Francesca Frascella,1,2 Annalisa \\ Chiappone1,2 \\ 1Dipartimento di Scienza Applicata e \\ Tecnologia, Politecnico di Torino, Turin; \\ 2PolitoBIOMed Lab, Politecnico di \\ Torino, Turin, Italy
}

\begin{abstract}
The 3D printability of cold-water fish gelatin used as co-initiating species for the crosslinking of PEGDA monomers in presence of camphorquinone (through a Norrish II reaction) is evaluated. The 3D digital light processing (DLP) leads to the creation of biocompatible printed structures able to support cell viability and proliferation.
\end{abstract}

\section{Introduction}

Recently, one of the main goals in bioengineering is the possibility to provide personalized scaffolds to resemble patient's tissue defects. Three-dimensional (3D) bioprinting can have the ambition to fulfill the needed requirements. ${ }^{1}$ In particular, Direct Light Processing (DLP) printers can create layer-by-layer models with high resolution and printing speed, regardless of the layer complexity and area. ${ }^{2}$ To be used as hydrogels, printable materials need to satisfy various criteria regarding printability, biocompatibility, and biomimetic properties, including mechanical and structural stability. Both synthetic and natural polymers were proposed to mimic the characteristic of extracellular matrix (ECM), but while natural polymers possess poor mechanical properties, synthetic polymers suffers of low biocompatibility. Herein cold-water fish gelatin is proposed as a new co-initiator for CQ in presence of PEGDA monomers ${ }^{3}$ to create a bioink where gelatin in fully incorporated into the PEGDA network.

\section{Materials and Methods}

Gelatin from cold-water Fish skin (CFG), poly(ethylene glycol) diacrylate (PEGDA, Mw 700), camphorquinone (CQ, 97\%),

Phenylbis $(2,4,6-$ trimethylbenzoyl)phosphine oxide (BAPO), and Brilliant Green (hydrogen sulfonate, BG). The formulation compositions are resumed in Table 1.

To characterize the reactivity of the system, photo-rheological tests were performed. To certify the incorporation of the gelatin into the PEGDA network, FT-IR and Insoluble fraction (IF) measurements were carried out, while amplitude sweep, DMTA and swelling kinetics test evaluated the mechanical performances of the hydrogel. Lastly on the 3D-printed geometries, MTT assays and phase contrast/fluorescence images were taken to prove the cells viability, cytotoxicity, and proliferation of the bioink.

\section{Results}

Firstly, the photo-rheological measurements proved the suitable reactivity of the system for DLP printing with a decrease of the reaction time and an increase of storage modulus with the gelatin content (in this stage two compositions were selected for further investigation, CFG15 and CFG50). Then some simple samples, 3D-printed both with CQ and BAPO photo-initiators, undergo to FT-IR and IF measurements confirming the chemical bonding of gelatin inside the PEGDA network by proving higher and sharper peaks of gelatin in the FT-IR and a higher IF (sign of the incorporation of gelatin) in the case of CQ-mediated Norrish II reaction. The mechanical properties of the hydrogel were tested through amplitude sweep, DMTA and swelling kinetics meas-
Correspondence:Michael Zanon, Dipartimento di Scienza Applicata e Tecnologia, Politecnico di Torino, C.so Duca degli Abruzzi 24, 10129 Turin, Italy.

E-mail: michael.zanon@polito.it

Key words: Hydrogel; DLP 3D printing; scaffold; gelatin.

Conference presentation: This paper was presented at the Third Centro 3R Annual Meeting - L'era delle 3R: modelli in silico, in vitro e in vivo per promuovere la ricerca traslazionale 30 September - 1 October 2021, Evento online organizzato dal Politecnico di Torino.

Received for publication: 9 July 2021.

Accepted for publication: 7 September 2021.

This work is licensed under a Creative Commons Attribution NonCommercial 4.0 License (CC BY-NC 4.0).

CCopyright: the Author(s), 2021

Licensee PAGEPress, Italy

Biomedical Science and Engineering 2021; 4(s1): doi:10.4081/bse.2021.201

urements, all confirming the improved characteristics with the increasement of gelatin quantity. Complicated geometries were then successfully 3D-printed (Figure 1) and use firstly to conditionate a medium to text the cells viability, cytotoxicity and proliferation. Cells were then seeded into the samples demonstrating the cell attachment and viability (Figure 2 ). In both cases, again, the increasements in gelatin content favored the biocompatibility of the bioink.

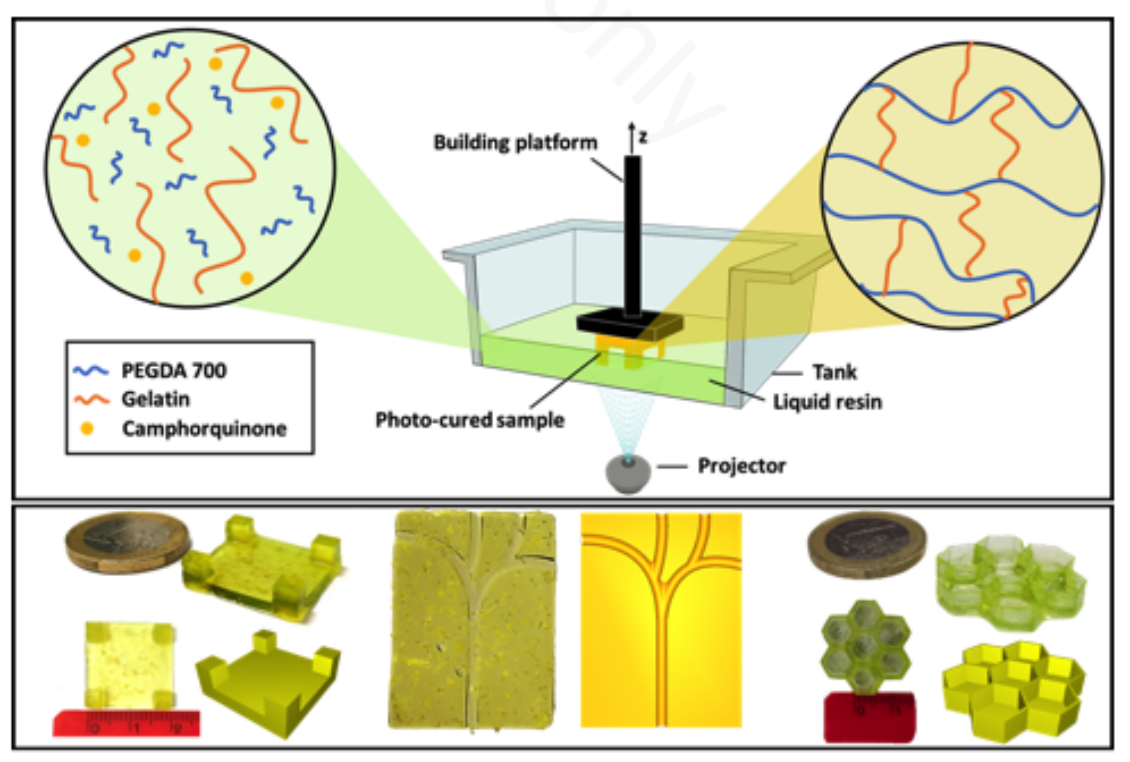

Figure 1. DLP scheme and printed geometries. 
Table 1. Formulation compositions.

\begin{tabular}{lccccc} 
& PEGDA $(w t \%)$ & WATHR $(w t \%)$ & CFG (phr)* & CQ (phr)* & BG (phr)* \\
CFG5 & 35 & 65 & 52 & 0.01 & \\
CFG10 & 35 & 65 & 10 & 2 & 0.01 \\
\hline CFG15 & 35 & 65 & 15 & 2 & 0.01 \\
CFG30 & 35 & 65 & 30 & 2 & 0.01 \\
\hline CFG50 & 35 & 65 & 50 & 2 & 0.01 \\
\hline
\end{tabular}

\section{Discussion and Conclusions}

In this study, gelatin was successfully incorporated into PEGDA network to form an interpenetrated hybrid system. Results showed improved mechanical and biological properties with the increase of the gelatin content. Furthermore, the implementation of this material leads to the creation of precise and rapidly printed structures that are biocompatible and promote cell viability and proliferation. Considering those features, the hydrogel may be a promising candidate for a 3D-printing bioink.

\section{References}

1. Murphy SV, Atala A. 3D bioprinting of tissues and organs. Nat Biotechnol 2014;32:773-85.

2. Kim SH, Yeon YK, Lee JM, et al. Precisely printable and biocompatible silk fibroin bioink for digital light processing 3D printing. Nat Commun 2018;9:1620.

3. Cosola A, Chiappone A, Martinengo C, et al. Gelatin type A from porcine skin used as co-initiator in a radical photoinitiating system. Polymers 2019;11:1901.

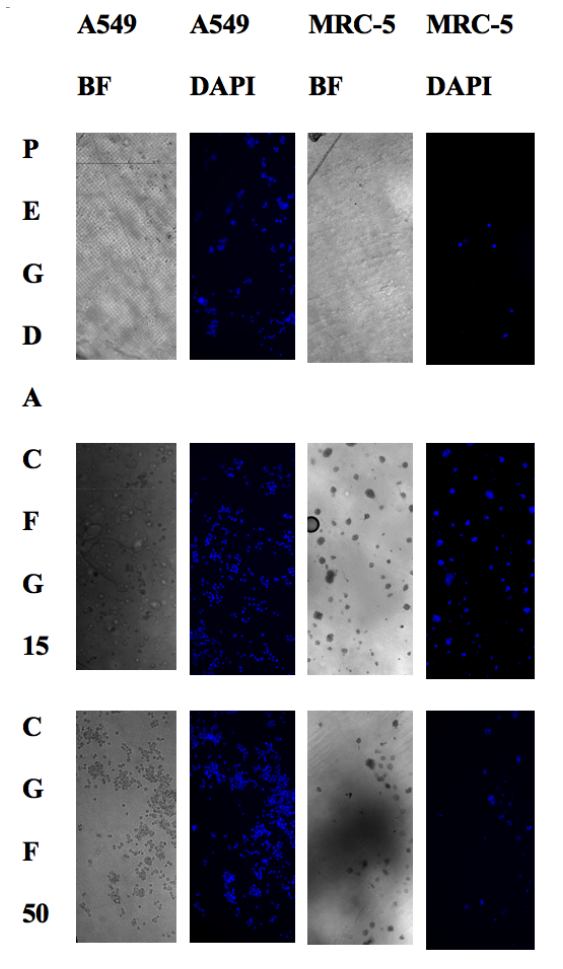

Figure 2. Phase-Contrast images and Fluorescence with two cell lines on PEGDA (used as control), CFG15 and CFG50. 